\title{
LETO (Littoral, État, Océan), un dispositif test sur les enquêtes littorales (XVII ${ }^{\mathrm{e}}-\mathrm{XIX}^{\mathrm{e}}$ siècles)
}

LETO (Coast, State, Ocean). A device under test on coastal surveys, 17th-19th centuries

\section{Martine Acerra}

\section{(2) OpenEdition Journals}

Édition électronique

URL : https://journals.openedition.org/abpo/4086

DOI : $10.4000 / a b p o .4086$

ISBN : 978-2-7535-7720-6

ISSN : 2108-6443

\section{Éditeur}

Presses universitaires de Rennes

\section{Édition imprimée}

Date de publication : 18 décembre 2018

Pagination : $69-90$

ISBN : 978-2-7535-7718-3

ISSN : 0399-0826

\section{Référence électronique}

Martine Acerra, «LETO (Littoral, État, Océan), un dispositif test sur les enquêtes littorales (xv॥lxIx ${ }^{\mathrm{e}}$ siècles) », Annales de Bretagne et des Pays de l'Ouest [En ligne], 125-4 | 2018, mis en ligne le 18 décembre 2020, consulté le 03 mars 2022. URL : http://journals.openedition.org/abpo/4086 ; DOI : https://doi.org/10.4000/abpo.4086 


\title{
LETO (Littoral, État, Océan), un dispositif test sur les enquêtes littorales (XVII ${ }^{\mathrm{e}}$-XIX ${ }^{\mathrm{e}}$ siècles)
}

\author{
Martine ACERRA \\ Professeur émérite en histoire moderne, université de Nantes - CRHIA
}

Dans le cadre d'un appel à projets du Contrat de Plan État Région (CPER) Pays de Loire ${ }^{1}$, une petite équipe pluridisciplinaire à dominante historique issue de plusieurs universités, régionales ou non ${ }^{2}$, a organisé en 2016 une série d'ateliers prospectifs ainsi qu'une journée d'études en 2017, afin de réfléchir à la mise en place d'un projet de recherche autour des enquêtes d'État concernant le littoral à l'époque moderne. Cette période constitue en effet un temps fort des rapports entre l'État, les villes portuaires et les économies maritimes ${ }^{3}$. L'action du pouvoir est visible, entre autres, au travers des enquêtes qu'il construit et diligente depuis le $\mathrm{XvII}^{\mathrm{e}}$ siècle jusqu'au début du XIX ${ }^{\mathrm{e}}$ siècle avant que se développe l'ère de la statistique. Qu'elles concernent l'ensemble du littoral français ou l'une de ses portions, qu'elles s'intéressent à un phénomène spécifique ou à un vaste panorama d'activités, ces enquêtes révèlent des moments et des orientations de la politique maritime française. Y apparaissent le souci de la présence de l'État sur mer comme sur les frontières côtières, la volonté du roi pour la domination du rivage et sa propension à tirer bénéfice des meilleures ressources économiques par une fiscalité pointilleuse.

1. Pour leur aide financière, logistique et scientifique, nous remercions le Contrat de Plan État Région des Pays de Loire, l'Institut Universitaire Mer et Littoral, l'UFR Histoire, Histoire de l'Art et Archéologie, les laboratoires de recherches CRHIA et LETG de l'université de Nantes et la MSH Ange Guépin.

2. Étaient représentées les disciplines suivantes : histoire (11), géographie (2), sciences économiques (2), droit (1), archéologie (1), informatique (1). Les universités concernées étaient Nantes, Lorient, La Rochelle, Bordeaux, Poitiers, Boulogne, Aix-Marseille, Paris-I Sorbonne.

3. La littérature abondante ne pouvant être citée, retenons pour exemple de l'intérêt pour cette thématique l'ouvrage collectif dirigé par LE BOUËDEC, Gérard et CHAPPE, François, Pouvoirs et littoraux du XV $V^{e}$ au XX $X^{e}$ siècle, Rennes, PUR, 2000. 
Certaines de ces enquêtes sont connues de longue date des historiens, d'autres font l'objet d'un intérêt récent. Nous y reviendrons. Mais quel que soit le cas, elles n'ont jamais été exploitées intégralement et collectivement du fait de leur ampleur, de leur complexité, de leur diversité. Aujourd'hui, grâce aux outils numériques, elles peuvent faire l'objet d'un traitement complet par décryptage, analyse et mise en perspective de leurs contenus, tant les préoccupations de l'époque moderne résonnent avec les préoccupations contemporaines concernant les littoraux : fragilité du milieu, effets climatiques et anthropiques, gestion et gouvernance y compris les conflits d'usage, entretien et exploitation responsable du domaine côtier comme des ressources halieutiques, pour ne citer que quelques exemples.

L'équipe LETO (Littoral, État, Océan) ne prétend pas apporter des solutions simples à ces problèmes complexes mais elle souhaite, par étapes prudemment menées, mettre à disposition de la communauté scientifique, des publics intéressés et des acteurs actuels de la gestion du littoral, les résultats de son travail collectif. Celui-ci passe par des outils à construire. La richesse informative des enquêtes littorales, qui sont massives et d'une grande variété formelle, exige la mise en place d'une plate-forme collaborative et pluridisciplinaire afin d'en extraire toutes les données géographiques, économiques, juridiques, environnementales. Sans posséder la précision et la rigueur des investigations du XxI ${ }^{\mathrm{e}}$ siècle, ces enquêtes permettent cependant d'envisager des représentations graphiques et des modélisations à comparer avec les mesures actuelles, afin de percevoir des évolutions sur le temps long. Face aux nombreuses difficultés de l'entreprise, l'équipe LETO a d'abord opéré par une phase exploratoire. Au cours des trois ateliers et de sa journée d'études, elle s'est donné comme objectif de réfléchir à une méthode de travail collective, d'en fixer les principes scientifiques et de mener des tests de faisabilité de son objectif final : fournir un site interactif de consultation des enquêtes et de l'analyse de leurs contenus, site envisagé comme une aide modeste mais réelle, par sa profondeur historique, aux prises de décision qui peuvent aujourd'hui concerner les littoraux ${ }^{4}$.

\section{Approche historiographique}

Pour comprendre comment l'équipe LETO a choisi de procéder et pourquoi, il convient d'abord de poser quelques jalons historiographiques concernant les enquêtes menées sur le littoral depuis l'époque de Richelieu, ce qui ne signifie pas, évidemment, que l'intérêt étatique pour cet espace naisse à ce moment-là. Sans remonter à l'époque même de la réalisation de ces enquêtes et à la bonne fortune que certaines ont pu connaître du

4. Rappelons le drame lié à la tempête Xynthia de 2010 et à la perte de la mémoire du bord de mer. Voir ACERRA, Martine et SAUZEAU, Thierry, " Zones construites, zones désertes sur le littoral atlantique. Les leçons du passé ", Norois, Xynthia, regards de la géographie, du droit et de l'histoire, $\mathrm{n}^{\circ}$ 222-2012/1, p. 103-114. 
fait de leur exploitation par des auteurs célèbres ${ }^{5}$, leur existence et leur contenu ont soulevé l'intérêt des historiens depuis la fin du XIX et le début du $\mathrm{Xx}^{\mathrm{e}}$ siècle.

Ainsi, Léon Maître publie en 1902 dans les Annales de Bretagne, un article sur l'enquête de 1664 organisée par Jean-Baptiste Colbert pour recenser toutes les embarcations constituant la flotte française d'alors. En 1941, Éric Dardel fait paraître aux Presses Universitaires de France un petit ouvrage sur les procès-verbaux des visites menées entre 1723 et 1737 par l'inspecteur des pêches Le Masson du Parc ${ }^{6}$. Dans son introduction, il indique un des aspects intéressants de cette vaste enquête thématique qui est de pouvoir mesurer la " profondeur de la mémoire d'État " sur ses connaissances littorales. Cependant, quelques décennies plus tard, ce point essentiel ne constitue pas la préoccupation majeure des auteurs qui s'emparent des enquêtes. Ils les utilisent surtout pour en extraire les données économiques et mesurer l'état du commerce français. Citons pour exemples les articles de Jean Delumeau et Michel Morineau parus dans la revue $X V I I^{e}$ siècle en 1966 et 1970, qui offrent une belle notoriété à l'enquête colbertienne de $1664^{7}$. Mais c'est à Jean Meyer que l'historiographie doit une nouvelle étape déterminante dans l'exploitation de ce type de documents. Dans sa thèse complémentaire sur l'armement nantais soutenue en 1966 et publiée en 1969, l'historien a l'idée, fort féconde, de confronter les informations issues de deux enquêtes réalisées à vingt ans d'intervalle ${ }^{8}$. Sont ainsi mobilisées pour illustrer le cas nantais, l'enquête de 1664 déjà citée et celle de 1683-1686 dite de Seignelay, réalisée afin d'évaluer la masse mobilisable des gens de mer français. Cette recherche fondatrice en matière de méthode quantitative a inspiré plus tard d'autres chercheurs qui, alors que se développaient les premiers outils de la micro-informatique, ont pris le relais en tentant de généraliser le procédé. André Zysberg est de ceux-là. Il a donné plusieurs livraisons des résultats de cette confrontation d'enquêtes appliquée à diverses portions du littoral français, sans pour

5. Nous pensons à l'exemple connu de Denis Diderot utilisant les mémoires et procèsverbaux de Le Masson du Parc. Voir : Pinault, Madeleine, "Diderot et les enquêtes de Le Masson du Parc ", dans BaLcou, Jean (éd.), La mer au siècle des encyclopédies, ParisGenève, Champion-Slatkine, 1987, p. 343-355; LIEPPE, Denis (éd.), Pêches et pêcheurs du domaine maritime et des îles adjacentes de Saintonge, d'Aunis, de La Rochelle et des Sablesd'Olonne. Procès-verbaux des visites faites concernant la pesche dans le ressort des amirautés de Marennes, de La Rochelle et des Sables-d'Olonne en 1727 et en 1728 [Le Masson du Parc], Saint-Quentin-de-Baron, éd. de l'Entre-Deux-Mers, 2009, 438 p.

6. MAITRE, Léon, «Situation de la marine marchande du comté nantais d'après l'enquête de 1664 ", Annales de Bretagne, t. 17, 1902-1903, p. 326-343; DARDEL, Éric, État des pêches maritimes sur les côtes occidentales de la France au début du XVIII siècle, d'après les PV de visite de l'inspecteur des pêches Le Masson du Parc (1723-1737), Paris, PUF, 1941.

7. DelumEAu Jean : "Le commerce extérieur français au XVII ${ }^{e}$ siècle ", Revue XVI ${ }^{e}$ siècle, 1966, p. 81-105; MORINEAU, Michel : « Flottes de commerce et trafic français en Méditerranée au XVII ${ }^{e}$ siècle ", Revue XVII siècle, 1970, p. 135-171.

8. MEYER, Jean, L'armement nantais dans la seconde moitié du XVIII siècle, Paris, SEVPEN, 1969. Pour l'identification de chaque enquête citée, nous renvoyons au tableau général des documents retenus pour les tests LETO, donné en fin de ce texte. 
autant qu'il y ait total dépouillement de l'enquête Seignelay. Tant il était difficile alors de mobiliser chercheurs et financements pour exploiter des documents si massifs.

Pour autant, l'intérêt des historiens pour les enquêtes littorales ne cesse de croître mais dans un contexte plus large de développement des recherches sur la formation des États modernes et de leurs moyens de gouvernement. Souvent utilisées en association avec d'autres types de documentation, les principales enquêtes des $\mathrm{XVII}^{\mathrm{e}}$ et XVIII ${ }^{\mathrm{e}}$ siècles livrent ainsi une part de leur richesse informative. Dans ces travaux, qui émaillent les vingtcinq dernières années, il est à noter qu'elles sont toujours étudiées dans un cadre local, pour une portion de littoral, une période spécifique ou une thématique particulière. Malgré l'absence d'étude globale, ces précieuses contributions enrichissent le débat historiographique sur la connaissance, la perception, l'utilisation des ressources civiles et militaires du littoral par ses acteurs locaux ou par l'État. L'excellent article d'Édouard Delobette consacré à la Normandie maritime illustre cette approche pour l'enquête de $1665^{9}$. Pour le " rolle de 1686 », nom que porte dans les archives de la chambre de commerce de Dunkerque un exemplaire de l'enquête dite de Seignelay, un des meilleurs connaisseurs est Christian Pfister-Langanay. Il en a publié les parties calaisiennes et dunkerquoises tout en l'utilisant plus globalement dans deux ouvrages sur Dunkerque ${ }^{10}$. Quant au littoral méditerranéen, théâtre de l'enquête de Séguiran en 1633, il bénéficie d'études présentées par Jacques Ferrier et plus récemment par Gilbert Buti, dans des colloques nationaux et internationaux ${ }^{11}$.

Point n'est besoin de multiplier à l'infini les exemples ni de chercher à dresser la liste exhaustive des publications ayant utilisé les enquêtes pour elles-mêmes ou comme type d'archives parmi d'autres, pour souligner leur place dans l'éventail des recherches actuelles sur le rôle du littoral dans le développement des économies européennes à l'époque moderne ou sur le rôle de l'État dans son appropriation multiforme des ressources côtières. Citons deux derniers exemples importants pour confirmer cette place avec la publication en cours de l'enquête Le Masson du Parc et avec l'enquête

9. Delobette, Édouard : "Les ports, havres et rivières navigables de Normandie dans l'enquête des amirautés de 1665 ", Annales de Bretagne et Pays de l'Ouest, tome 121, n 4, 2014, p. 39-80.

10. Pfister-Langanay, Christian, " Un recensement de la flotte de Calais en 1686 », Bulletin Historique et Artistique du Calaisis, $\mathrm{n}^{\circ}$ 87, 1982, p. 182-188; " La flotte dunkerquoise en 1686 ", Revue Historique de Dunkerque et du Littoral, n 37, janvier 2004, p. 413-429; Ports, navires et marchandises à Dunkerque, 1660-1792, Dunkerque, 1985; Constructeurs, chantiers et navires à Dunkerque du XVII au XX $x^{e}$ siècle, préface de J.-P. Poussou, Dunkerque, Société dunkerquoise d'histoire et d'archéologie, 2005, 434 p.

11. FERRIER, Jacques, "La Méditerranée, mer ouverte à la France : le tournant historique de 1633 " dans VilLain GANDOSsi et al. (dir.), Méditerranée, mer ouverte, 2 vol., tome 1, Malta, 1997, p. 63-72; BuTı, Gilbert, "La flotte de commerce et de pêche de la France méditerranéenne à la fin du XVII ${ }^{e}$ siècle d'après les enquêtes de Colbert et Seignelay ", dans Tourville et les marines de son temps, Saint-Lô, 2003, p. 49-90. 
Chardon menée entre 1781 et 1785, objet de l'HDR de Sylviane Llinarès soutenue en 2011 et dont la publication est attendue ${ }^{12}$.

Ainsi, depuis environ une décennie, des historiens sensibles au gisement informatif de ces enquêtes ont réfléchi, seuls ou collectivement, aux moyens de les exploiter ${ }^{13}$. Parmi eux, plusieurs membres du présent dispositif LETO ont discuté de façon informelle avant de se lancer dans l'opération dont résultent les textes publiés en varia dans le présent numéro des Annales de Bretagne et Pays de l'Ouest et dans le suivant. Après quelques constats sur l'état de l'art et les possibilités numériques d'aujourd'hui, il est ressorti des échanges des choix méthodologiques afin de répondre à quelques objectifs.

\section{Choix méthodologiques}

Aborder les enquêtes littorales dans leur continuité séculaire, du règne de Louis XIII au début de la Restauration, résulte d'un choix volontariste. Il permet d'évaluer les évolutions selon deux grands axes d'analyse complémentaires. Le premier concerne le décryptage de l'art de l'enquête littorale et de toutes ses facettes sur la longue durée. Le second consiste à dresser le bilan des connaissances recherchées et accumulées sur le littoral, avec les variantes et effets de miroir d'une période à une autre. Pour ce faire, la série des enquêtes sélectionnées est complétée par des groupes iconographiques retenus pour leur pertinence et leur proximité chronologique des premières. Nous les décrivons plus loin. Ponctuellement, d'autres documents périphériques ont été convoqués afin d'éclairer certains aspects obscurs des enquêtes. L'ensemble ainsi constitué pourra être ultérieurement complété. Il permet d'ores et déjà d'envisager la création de deux outils collaboratifs : un site internet et un SIH (système informatique historique) inspiré des SIG construits par les géographes, dont la méta-base regroupant les enquêtes sera le cœur ${ }^{14}$.

12. LiePpe, Denis, Pêches et pêcheurs..., op. cit. ; LlinAREs, Sylviane, Autour de l'inspection Chardon, 1781-1785. Enquête, réforme et modernisation. Les amirautés et la politique maritime de la France sous Louis XVI, HDR soutenue le 10 décembre 2011, Université de Bretagne-Sud.

13. Avant de faire l'objet des trois ateliers financés par le CPER (Contrat de Plan État Région) Pays de Loire, la réflexion collective a été présentée dans une communication lors du II ${ }^{\mathrm{e}}$ Congrès du GIS Histoire maritime (aujourd'hui d'Histoire et sciences de la mer) tenu à Nantes en juin 2013, dont nous reprenons certains éléments méthodologiques. Cette contribution a été récemment publiée : ACERRA, Martine, BuTI, Gilbert, LLINARES, Sylviane, PFISTER, Christian, "Inspecter le littoral français à l'époque moderne ", dans La maritimisation du monde, Paris, PUPS, 2016, p. 497-513. Par ailleurs, depuis 2015 Sylviane Llinarès a initié un groupe de travail autour de l'enquête Chardon et de la mise à disposition publique des PV de visites. Plusieurs chercheurs de ce groupe participent aussi au dispositif LETO.

14. Les exemples de mise en ligne de travaux collaboratifs ne manquent pas. En histoire maritime, il peut s'agir du site navigocorpus.org, né du financement ANR (2007-2011) dirigé par Silvia MARZAGalli, Pierrick PouRChASSE et Jean-Pierre DEDIEU. Il propose à la consultation le corpus des itinéraires de navires de commerce du XVII ${ }^{\mathrm{e}}$ au XIX ${ }^{\mathrm{e}}$ siècle. Dans un autre 
L'équipe LETO n'a pas vocation à réaliser l'ensemble des opérations nécessaires pour atteindre ces objectifs car la tâche est immense. Par exemple, la transcription de milliers de folios représente à elle seule un travail de longue haleine qui devra être mené selon des méthodes rigoureuses respectées par tous. Cette transcription ne pourra donc nourrir que progressivement la méta-base envisagée. Par ailleurs, les enquêtes littorales, seules ou associées à diverses documentations, font l'objet d'autres programmes en cours ou travaux individuels que le dispositif LETO n'entend pas concurrencer ${ }^{15}$. Au sein même de l'équipe, chacun est libre de préserver sa recherche personnelle. Les objectifs de chacun s'avèrent souvent complémentaires. Dans cet esprit, le dispositif LETO souhaite avant tout bâtir un cadre de réflexion où chacun peut, s'il le souhaite, apporter sa contribution et utiliser ce qui aura été mis à disposition de tous.

L'ensemble documentaire retenu pour tester la future méta-base est constitué de neuf enquêtes, deux groupes archivistiques et huit corpus iconographiques pour la période 1629-1811. Les enquêtes couvrent plus ou moins l'ensemble du littoral et, en ce domaine, forment trois catégories. Les premières, que l'on peut qualifier de globales ou générales même si elles comportent des lacunes, concernent les deux façades maritimes du royaume. Les deuxièmes n'intéressent que le Ponant ou le Levant et les troisièmes un secteur d'un de ces littoraux.

Dans le contexte évoqué précédemment de la construction de l'État moderne, il n'est pas étonnant que le XVII ${ }^{\mathrm{e}}$ siècle soit plus émaillé d'enquêtes que le siècle suivant au cours duquel les moyens administratifs de l'État augmentent et assurent une régulière information du pouvoir, avant que les nécessaires réformes maritimes et navales ne s'imposent dans les dernières décennies pré-révolutionnaires. Les règnes de Louis XIII et Louis XIV correspondent à une frénésie certaine de l'inspection littorale et voient la réalisation de deux enquêtes diligentées par Richelieu (Le Roux d'Infreville, 1629, au Ponant; Séguiran, 1633, au Levant), partielles mais complémentaires en termes territoriaux. Puis viennent celles ordonnées par Louis XIV et suivies par les différents secrétaires d'État qui se succèdent au département de la Marine entre 1664 et 1698 (Inventaire général des vaisseaux... 1664; Havres et ports de France, 1665; Rôle général des bâtiments de mer, 1683-1686, toutes trois pour l'ensemble du littoral; Mémoire de Béchameil de Nointel, 1698, pour la Bretagne seule).

registre et en association avec le Service Historique de la Défense de Cherbourg, le CRHQ (Centre de Recherche en Histoire Quantitative) de l'Université de Caen-Basse Normandie a mis en ligne le programme CIMARCONET recensant l'inscription maritime du Cotentin d'après les registres matricules (unicaen.fr/ufr/histoire/cimarconet).

15. Parmi les programmes en cours, peuvent être cités le groupe de travail, déjà évoqué, sur l'enquête Chardon piloté par Sylviane Llinarès ; celui organisé conjointement par Gauthier Aubert, Philippe Jarnoux et Pierrick Pourchasse concernant la Bretagne, auteurs de la réédition des mémoires de Colbert de Croissy et de Béchameil de Nointel; le programme DYPOMAR, outil interactif traitant de trois thématiques et associant données manuscrites, cartographiques, iconographiques. 
Puis, au silence décennal des archives met fin l'enquête sur les pêches confiée à l'inspecteur Le Masson du Parc qui parcourt le Ponant pour l'exécuter entre 1723 et 1737 . Outre celle-ci, dont s'inspire pour partie l'enquête générale de Chardon menée entre 1781 et 1785 , nous avons retenu l'enquête de l'ingénieur Perronet de 1770 concernant la Loire car sa précision annonce l'ère de la bathymétrie scientifique. Enfin, la grande enquête de 1811 commandée par le ministère de l'Intérieur sur le commerce et les moyens de transport termine notre sélection. Les deux groupes d'archives, que nous avons choisis d'intégrer pour reconstituer une sorte de continuité documentaire, touchent pour l'un au pilotage en Gironde dans la seconde moitié du XVIII ${ }^{\mathrm{e}}$ siècle et pour l'autre le Département maritime de Nantes en $1774^{16}$.

Quant à l'iconographie sélectionnée, elle n'est pas convoquée au simple titre d'illustration, mais comme véritable outil informatif d'aide à la décision. Certaines enquêtes contiennent des figurations, telle celle sur les havres et ports de France de 1665. D'autres sont à l'origine de recueils de cartes et plans. Une autre catégorie constituée d'albums, factices ou non, permet de préciser les contenus techniques des enquêtes, tel que la typologie des navires pour ne citer que ce cas. L'iconographie est donc un des éléments majeurs dont disposaient les commanditaires de l'époque moderne pour se forger leur connaissance du littoral et y décider d'aménagements au bénéfice des navigateurs comme de l'État ${ }^{17}$.

Les corpus iconographiques retenus appartiennent à plusieurs catégories, techniques et supports. Ils sont tous accessibles soit dans les fonds où ils sont conservés, soit dans les musées ou sous forme de publications. Pour le XVII ${ }^{\mathrm{e}}$ siècle, il s'agit de trois albums, tous publiés, représentant des embarcations de mer ainsi que la construction d'un vaisseau de guerre : l'album dit de Colbert (circa 1670) et les deux dits de Jouve de 1679. S'y ajoutent deux recueils de cartes et plans des années 1680-1690 conservés au Service Historique de la Défense. Le XviII ${ }^{\mathrm{e}}$ siècle bénéficie, quant à lui, d'un remarquable recueil factice de dessins illustrant les pêches côtières et lié à l'inspection de Le Masson du Parc ${ }^{18}$. Tout aussi remarquables pour leurs qualités informatives et esthétiques sont les tableaux de Joseph Vernet sur les ports de France et les séries de gravures et d'estampes des frères Ozanne.

16. Les archives sur les pilotes de la Gironde ont été réunies par Jacques Péret.

17. On peut citer comme preuve de l'importance de la cartographie l'existence du recueil SH 79 (en dépôt au SHD Vincennes), relié aux armes des Colbert, né, selon E. Delobette ("Les ports, havres... ", art. cit., p. 46), de l'ordre du roi à Seignelay en 1676 pour améliorer la sécurité de la navigation côtière.

18. Il s'agit de l'ensemble conservé à la BnF, cabinet des estampes, Ke-82 fo, vestiges des archives dispersées de Le Masson du Parc. Voir aussi : PinAult, Madeleine, "Diderot... ", art. cit.; LIEPPE, Denis, Pêches et pêcheurs..., op. cit. 


\section{Problématiques et champs de recherche}

Dans la longue histoire du littoral, le développement du processus de l'enquête marque une étape décisive quant à la connaissance de cet espace à la fois territoire et frontière vivante, productrice, stratégique. Il souligne aussi l'intérêt croissant de l'État ainsi que sa capacité, qu'il construit progressivement au cours de l'époque moderne, à s'informer. La décision d'organiser une enquête littorale à l'échelle du royaume, telle qu'elle apparaît avec celles de 1664 et 1665, constitue d'ailleurs, en soi, une étape intellectuelle d'importance et le signe d'une maturité politique certaine par la globalité de sa perception du double littoral français. Le processus de l'enquête analysé à l'échelle bi-séculaire, comme l'envisage le test LETO, doit permettre de saisir les convergences et les différences qui existent d'un cas à l'autre, autant dans les contenus mêmes que dans la méthode suivie.

Les approches historiques peuvent en varier selon les centres d'intérêt de chaque chercheur, surtout s'il associe à sa démarche les apports d'autres disciplines telles que la géographie, le droit ou les sciences économiques. Il vient à l'esprit des thématiques classiques telles que les évolutions de la flotte de commerce, le développement économique et structurel des villes-ports ou la fiscalité royale pour ne citer que ces cas, qui peuvent être traités selon un jeu d'échelles allant du micro-local au national.

Mais la comparaison des contenus informatifs, qui est l'un des deux axes principaux d'exploitation des enquêtes qu'envisage le test LETO, fait apparaître une série d'autres études possibles, inscrites dans le cadre général des ressources et du contrôle du littoral. Traitées différemment d'une enquête à l'autre, elles n'en constituent pas moins les lignes directrices des préoccupations de l'État commanditaire ${ }^{19}$. Parmi ces grandes orientations, les activités maritimes se distinguent évidemment, qu'il s'agisse de la pêche, du commerce, de la construction navale et de leurs flottes dédiées. Ces activités sont directement connectées au thème de la sûreté maritime nécessaire dans les ports, sur les côtes, en mer. À cette occasion apparaissent le souci étatique pour les aménagements du littoral et son goût pour le contrôle, la surveillance, la protection de sa frontière maritime. Contrôle qui s'exerce également par le biais de la fiscalité, thème récurrent de la plupart des enquêtes quelle que soit la façon dont il est abordé. Les ressources humaines et matérielles font aussi l'objet de questionnements précis dans le cadre de préoccupations économiques plus vastes.

Du XVII ${ }^{\mathrm{e}}$ au XIX ${ }^{\mathrm{e}}$ siècle, les enquêtes disposent de moyens différents et usent de modalités spécifiques mais elles visent un certain nombre d'objectifs communs. Par ailleurs, l'analyse comparative permet de mesurer la façon dont l'État maîtrise progressivement l'énoncé de ses objectifs afin d'en obtenir une exécution rigoureuse par les destinataires auxquels il

19. Notons cependant que certaines enquêtes sont ciblées sur un phénomène ou orientées sur quelques thématiques. Mais il est toujours possible pour le chercheur de mobiliser conjointement plusieurs enquêtes pour étudier l'évolution du phénomène concerné. 
s'adresse. Nous abordons ici une autre série d'études rendues possibles par la prise en compte globale des corpus documentaires et iconographiques retenus. Apparaît ainsi l'évolution séculaire de la façon dont l'État expertise et s'informe, qui peut se scinder en trois temps : avant, pendant, après l'enquête. En ce sens, "à l'époque moderne, le processus de l'enquête s'apparente à une sorte de cadre programmatique qu'il convient de rapprocher des sept niveaux nécessaires à la décision, utilisés dans les études contemporaines de sciences politiques ${ }^{20}$ ".

À l'origine d'une enquête, il est toujours un constat, directement formulé ou non, né d'événements plus ou moins anciens affectant le littoral et dont s'inquiète l'État. Ainsi, pour l'enquête de 1665 sur les havres et ports de France, l'arrêt du Conseil qui ordonne l'inspection des côtes fait-il référence à la détérioration de la navigabilité portuaire par le délestage intempestif des maîtres de navire. Les tournées et visites de l'inspecteur des pêches Le Masson du Parc (1723 à 1727) sortent de la raréfaction constatée de la ressource halieutique consécutive, selon un intendant de l'époque, à l'inobservation de l'ordonnance de marine de 1681 et à la présence de nombreuses pêcheries côtières ${ }^{21}$. Menées sur un vaste secteur du littoral, les visites de Le Masson du Parc ne sont pas les premières à s'intéresser à certaines portions de la côte régulièrement observées telles que la Picardie, les Flandres, la Normandie. Mais elles en diffèrent par la mission confiée qui est la première ciblée sur un objet unique, les pêches.

Pour toute enquête, il convient non seulement de déterminer son origine et les modèles dont elle a pu s'inspirer, mais aussi les motifs de sa commande, la façon dont ils sont exprimés grâce à une " feuille de route " plus ou moins détaillée, et à quels exécutants elle est confiée. L'analyse de la préparation des enquêtes prête aussi à de nombreux constats. Méthode, cadre juridique, périmètre d'exécution varient mais il est possible d'y déceler des constantes, une meilleure définition et une progression des exigences étatiques. L'ordre royal peut être accompagné et renforcé d'une liste de points à exécuter, sorte d'instructions complémentaires sous formes de questionnaires ou de mémoires exigeant d'observer certains aspects précis. Pour les enquêtes de 1664 et 1665 suivies par Colbert, celui-ci est l'auteur du questionnaire accompagnateur. Sous le prétexte de réguler les excès du délestage sauvage et s'appuyant sur l'arrêt royal du 24 janvier 1665, Colbert déroule une liste de trente-huit questions visant à fixer ce qu'est exactement un " havre recommandable ", dévoilant ainsi ses vastes préoccupations maritimes destinées, entre autres, à la reprise en main du littoral et à l'implantation d'un nouvel arsenal au Ponant ${ }^{22}$. Les lettres de

20. Acerra, Martine, Buti, Gilbert, Llinares, Sylviane, Pfister, Christian, « Inspecter le littoral français..." ", art. cit., p. 503.

21. DARDEL, Éric, État des pêches maritimes..., op. cit., p. 5. Référence au mémoire de l'intendant de Caen en date du 21 septembre 1723 (Archives départementales du Calvados, C4/145).

22. SHD Vincennes, SH 48, Instruction envoyée à tous lesdits officiers pour leur dire la manière de faire ladite visite afin de connaître les bonnes et mauvaises qualités des ports. 
commission destinées à chaque inspecteur ainsi que les éventuelles instructions complémentaires offrent donc de précieuses pièces pour l'étude de la mise en place et du rôle de la décision politique. Il est également à noter que l'État confie l'exécution de ses ordres d'inspection à des acteurs différents, exerçant dans des cadres juridiques variés. Globalement, l'exercice de l'enquête se décline en deux grandes techniques d'investigation. L'inspection peut être confiée aux institutions et structures administratives présentes sur le terrain. Il peut s'agir des amirautés et des justices locales comme dans le cas des enquêtes de 1664 et 1665; du commissariat des classes pour celle dite de Seignelay des années 1683-1686 ou de celle partielle de 1774 pour le quartier de Nantes. L'autre technique d'investigation consiste à recourir à des envoyés spéciaux, des inspecteurs commis à cet effet, qui agissent sur le terrain, seuls ou accompagnés, avec ou sans référents locaux dont les statuts socio-professionnels sont également variables. Richelieu envoie Le Roux d'Infreville sur les côtes du Ponant et Séguiran sur les méditerranéennes. Louis XIV s'en remet à Colbert de Croissy pour une enquête particulière sur la Bretagne menée en 1665, et à Béchameil de Nointel en 1698 pour la même province. Le Masson du Parc et Chardon sont des inspecteurs de terrain agissant selon des méthodes différentes, le second s'appuyant sur un long travail préparatoire assuré par les cinquante et un sièges d'amirauté qu'il visite ensuite. D'une autre catégorie professionnelle, l'ingénieur des Ponts et Chaussées Péronnet assure son inspection de 1770 dans des conditions spécifiques. La comparaison de toutes ces modalités de commande et d'exécution est riche d'enseignements et autorise nombre de constats sur les acteurs de l'enquête et leur rôle avant même le début de celle-ci. La comparaison permet aussi de mesurer le degré d'aisance et de moyens que développe l'État pour faire exécuter ses ordres.

L'étape de réalisation des enquêtes permet d'autres orientations de recherche qui touchent aux conditions matérielles, techniques et humaines de leur exécution par des responsables en place ou des inspecteurs commis à cet effet. Selon les cas, le temps de réalisation est très variable. Il peut être très rapide, de l'ordre de trois mois comme dans le cas des visites de 1665 sur les havres et ports de France. Mais il peut aussi se compter en années, ainsi qu'il en est pour l'inspection de Le Masson du Parc qui l'effectue en une dizaine de tournées et visites, repassant parfois par les mêmes lieux entre 1723 et 1737 . Il faut aussi plusieurs années à Chardon pour accomplir sa vaste inspection entre 1781 et $1785^{23}$.

Dans la pratique même de la visite et quel que soit l'exécutant responsable, le temps consacré à un lieu sur le terrain diffère selon des critères et pour des motifs qu'il conviendra de comprendre. Durant d'une à quinze

23. L'inspection du commissaire du roi Daniel Chardon commence en 1781 avec les amirautés de Bretagne, puis viennent celles de Flandres et de Picardie l'année suivante, en 1783 celles de Normandie, Poitou, Aunis, Saintonge et Guyenne. L'inspection s'achève en 1785 avec les amirautés de Méditerranée, Languedoc, Roussillon, Provence et Corse. 
journées et parfois plus, la visite peut être minutieusement décrite dans ses moindres détails ou, au contraire, rester muette sur les conditions de déplacement local, laissant supposer que, parfois, aucun responsable ne quitte sa résidence officielle pour exécuter les ordres royaux. Lorsque le détail abonde, il est possible de tracer l'itinéraire de l'inspection ou de le décomposer grâce à la cartographie d'époque. Ainsi, lorsque, en 1665, le personnel de l'amirauté de Guyenne inspecte l'estuaire de la Gironde en le parcourant de Bordeaux à la mer et retour sans négliger le cours de la Dordogne jusqu'à Libourne, la confrontation des détails du procès-verbal de visite avec les données fournies par la carte de l'embouchure comprise dans le recueil SH 79 permet de visualiser exactement quand et où se trouve la petite troupe, pour quelles raisons (nuit, fort courant, vent contraire, marée insuffisante) elle interrompt ses opérations, où elle fait étape pour passer la nuit, à quel endroit elle passe d'une rive à l'autre de l'estuaire, etc. ${ }^{24}$. Ce genre de reconstitution et de compréhension du terrain est possible dans nombre de cas et justifie pleinement le recours à l'iconographie originale aux côtés des procès-verbaux de visites.

Le temps de la réalisation des enquêtes est aussi l'occasion de saisir une autre richesse de cette documentation. Elle fourmille d'informations sur différents acteurs du littoral. Il s'agit des enquêteurs eux-mêmes bien sûr, mais aussi des " experts " convoqués et des populations décrites par divers biais, même si nombre d'enquêtes s'avèrent incomplètes ou lacunaires sur ce point. Les personnels officiels requis pour l'inspection méritent l'attention. Représentants de diverses institutions ou fonctions, leur étude à plusieurs moments de la période moderne participe de l'histoire administrative de l'État pour laquelle les visites de Chardon présentent un remarquable cas de figure, dans la mesure où elles relèvent autant de l'enquête sur le littoral que de l'enquête sur les amirautés elles-mêmes. Mais la richesse humaine des corpus retenus gît également dans la masse des personnages qui apparaissent incidemment au cours des tournées officielles. Pour répondre aux objectifs de l'enquête et à l'éventuelle feuille de route qui les complète, les responsables de l'inspection réquisitionnent les savoirs locaux. Ils convoquent donc tous ceux qui sont susceptibles de connaissances et capables de les exprimer. Encore une fois, plusieurs cas de figure existent, de la convocation nominale à l'allusion globale à un groupe de " bourgeois habitants ", de " connaissants ", de pilotes, marchands, délesteurs, etc.

24. La visite de l'estuaire commence le 16 avril 1665 à Bordeaux où l'équipe de huit personnes embarque à bord d'une chaloupe. Le retour a lieu le 26 avril suivant. L'itinéraire suit d'abord la rive saintongeaise jusqu'à Blaye où l'inspection patiente et dort à cause des vents contraires. Elle traverse le fleuve le lendemain pour rejoindre la côté médocaine à Pauillac. Un nouvel arrêt a lieu à Mapon le 19 avril « ne pouvant aller à force de rames contre le flot à cause de la rapidité des courants ". Puis ce sont les étapes de la Goulée et du Verdon avant le contournement du phare de Cordouan, la reconnaissance des hauts fonds et les étapes à Saint-Palais, Plassac; la remontée de la Dordogne; les arrêts à Libourne et Bourg avec consultation des pilotes locaux. Le dernier tronçon d'inspection longe le bec d'Ambès et fait revenir l'équipe à Bordeaux. 
Parfois, les procès-verbaux sont muets sur ce point; parfois ils regorgent de détails, y compris à l'intérieur d'une même enquête. Dans ce cas, lorsque le rédacteur procède avec rigueur et précision, il fournit à l'historien une précieuse masse d'informations sur une partie de la population littorale ainsi sortie de l'ombre des archives. Lors de sa visite du cours et des rives de la Gironde qui dure onze jours, le lieutenant général de l'amirauté de Guyenne convoque successivement des pilotes de Bordeaux, Pauillac, Libourne et Bourg dont les nom, prénom, âge et résidence sont indiqués. Il se fait accompagner d'eux lors de son parcours fluvial pour qu'ils l'aident à saisir les dangers de certains passages, les avantages de quelques mouillages, la " tyrannie du tirant d'eau ${ }^{25}$ " lorsqu'à divers endroits les fonds sont insuffisants, les risques de submersion liés aux grandes eaux de mars et aux fortes marées provoquant le célèbre mascaret. Ces précieux auxiliaires, aux compétences reconnues, sont âgés de 38 à 63 ans; des " jeunes " comparés aux trois " bateliers, délesteurs et habitants de Bordeaux " âgés de 65, 75 et 78 ans, appelés à témoigner pour leur mémoire des temps anciens où le port de la Lune n'était pas encore encombré des détritus qui en avaient depuis dégradé la navigabilité.

Enfin, au cours de certaines inspections telles que l'enquête de 1664 sur la flotte française, de 1683-1686 sur les navigations ou de Le Masson du Parc de 1729 et 1730, sont nommées d'autres catégories de populations littorales : les maîtres de barque et de navire, les armateurs, les capitaines mais aussi les plus modestes des pêcheurs. Ainsi l'inspecteur des pêches décrit ces petits acteurs côtiers et leurs misérables cabanes adossées à la dune ou perdues dans le marais. L'inspecteur est connu pour cette particularité de dresser lieu par lieu, la liste des pêcheurs riverains, avec leur nom, prénom, âge, profession, offrant ainsi à l'analyse un riche gisement documentaire sur nombre de silencieux de l'histoire. Et, si les femmes en sont absentes, le recueil factice de dessins liés à cette inspection les fait apparaître, accompagnées des enfants, dans leurs tâches de cueillette et de pêche littorales comme autant de réelles actrices d'une économie maritime de survie.

Le troisième temps de l'inspection du littoral, celui de l'après enquête, mène à de nombreuses interrogations quant à son utilité réelle, immédiate ou différée; à son efficacité à répondre aux objectifs fixés; à ses réceptions, résonances, conséquences pour les populations et institutions locales. Les enquêtes, telles que nous les avons sélectionnées, semblent pour la plupart accompagnées d'investigations préalables ou parallèles, parfois massives, qui fournissent par elles-mêmes des éléments de réponse. Le rôle de ces tournées littorales complémentaires mérite d'être associé à l'analyse de l'impact réel des enquêtes, tant il élargit notre connaissance des processus étatiques. Il en est de même des documents préparatoires à la rédaction finale des mémoires et procès-verbaux officiels, ainsi que des lettres et des demandes complémentaires qui ont pu suivre l'inspection.

25. Expression empruntée à Thierry Sauzeau. 
Cet ensemble documentaire permet de cerner l'efficacité de l'enquête par rapport aux motivations de l'État commanditaire, comme il révèle également les éléments constitutifs d'une vision par le haut du monde littoral et maritime. Celle-ci naît d'une accumulation de connaissances acquises au cours des décennies dont le rythme et l'amélioration peuvent être mesurés grâce à l'analyse de la " mémoire d'État " sur le temps long. Peut-on déceler l'utilisation des acquis d'une enquête à l'autre? Si oui, quels en sont les vecteurs? Quelles sont les références citées, implicites, soupçonnables qui lient les inspections successives? Enfin et surtout, quels résultats concrets émanent de ces enquêtes, avec ou sans temps de latence nécessaire à la " digestion " de leur masse informative?

Des recherches récentes apportent quelques réponses et annoncent celles à venir ${ }^{26}$. Ainsi, la continuité mémorielle de l'État peut tenir à la longévité d'acteurs de terrain, tel Régnier Jansse de Wit fils qui exerce ses fonctions successives au service du roi pendant plus d'un quart de siècle. Il apparaît aux côtés de Le Roux d'Infreville chargé de l'inspection du Ponant en 1639. Vingt-cinq ans plus tard, un dessin d'écluse, daté et signé de sa main, se trouve inséré à la suite du procès-verbal de la visite du Cap de Cette effectuée en exécution des lettres patentes du 25 janvier 1665 ordonnant l'inspection des havres et côtes de France ${ }^{27}$. Dans le mémoire accompagnant ce dessin, il se désigne comme " ingénieur ordinaire du roi pour les dighues et travaux de la mer ». Ce simple cas autorise plusieurs remarques. Un modèle d'écluse d'origine hollandaise circule de ponant en levant grâce à un ingénieur possédant une réelle mémoire des lieux et une compétence professionnelle reconnue. L'inspection de 1665 est l'occasion de ce transfert. Par ailleurs, elle est accompagnée d'autres missions de terrain ayant pour objectif la compréhension du littoral et son aménagement. Ainsi, un groupe de fidèles de Colbert - son cousin Colbert de Terron, le chevalier de Clerville, l'ingénieur Chatillon, l'architecte François Blondel et, encore, Régnier Jansse - effectue une tournée des côtes de France afin de trouver un lieu propice à la relâche des vaisseaux de guerre. Le contexte international et la prudence de Colbert justifient cette technique de la double enquête qui prouve le souci du futur secrétaire d'État à la Marine de s'émanciper de réponses officielles et peut-être convenues des amirautés. L'une et l'autre inspection de 1665, qu'il faut coupler avec celle de l'année précédente sur la flotte commerciale du royaume, déterminent les choix de l'État en matière

26. Nous reprenons en les synthétisant une partie des réflexions de l'article collectif : ACERRA, Martine, ButI, Gilbert, LlinAREs, Sylviane, PfISTER, Christian, " Inspecter le littoral français... ", art. cit., p. 508-510

27. SHD Vincennes, manuscrit SH48, dessin non folioté suivant un mémoire du 18 juillet 1665 signé de Régnier Jansse, inséré f ${ }^{\circ} 251$ recto et verso, en suite au procès-verbal de visite du Cap de Cette, fo 243 à 250 v $^{\circ}$. Un encart en bas à gauche du dessin porte la mention suivante : "Modelle de l'escluse faict cy devant a Dunquerque qu'il faudra faire a soixante dix thoises du rivage de la mer dans le canal qu'il fault creuser au cap de Cette pour joindre lestang et pour il faire passer les barques faict a Monpellier le $16^{\mathrm{me}}$ juillet 1665 Regnier Jansse ". 
d'implantations portuaires pérennes. Le temps de réponse aux informations collectées est fort court puisque les décisions royales sont prises en 1666. Après quelques hésitations, le cours de la Charente et la terre de Rochefort sont retenus pour l'édification d'un arsenal. Le site de Lorient voit la fondation d'un port dévolu à la compagnie des Indes Orientales tandis que le port de Sète est créé au débouché du canal du Midi. Il n'est pas douteux que la détermination de ces trois lieux choisis pour des fonctions différentes et complémentaires sort de la connaissance acquise des enquêtes évoquées ci-dessus. Elles ont d'ailleurs d'autres conséquences à plus long terme, non plus structurelles mais administratives et fonctionnelles. Sujet de polémiques et de conflits d'usage, le délestage intempestif à l'origine de l'inspection de 1665 débouche sur des réglementations énoncées dans les titres et articles de l'ordonnance de 1681 où s'expriment les normes à respecter en ce domaine ${ }^{28}$. Les questions précises de Colbert dont la liste accompagne les lettres patentes du 25 janvier 1665 trouvent aussi leur écho dans nombre de titres et articles de l'ordonnance de 1689 sur les arsenaux et les armées navales. Même si cette dernière doit par ailleurs beaucoup aux mémoires préparatoires des techniciens de la marine militaire.

$\mathrm{Si}$ au XVII ${ }^{\mathrm{e}}$ siècle les enquêtes participent à l'élaboration de la politique maritime du royaume, au siècle suivant, l'inspection de Daniel Chardon s'insère exactement dans le cadre des réformes de la navigation et du commerce maritime de la fin du règne de Louis $\mathrm{XVI}^{29}$. Le cas de figure semble exceptionnel puisque les réformes en cours s'inspirent et s'enrichissent des observations faites au jour le jour sur le terrain, dans une sorte d'immédiateté, en réalité largement facilitée par les échanges préparatoires à l'inspection. La décennie, qui contient les années de l'enquête Chardon (1781-1785) ainsi qu'une floraison de mémoires et rapports sur l'état du fait maritime en France, est aussi celle de la formulation d'un vaste programme touchant les affaires maritimes, la protection du commerce, l'administration portuaire. Les procès-verbaux de l'inspection nourrissent incontestablement les réflexions et décisions en cours.

Quant à la réception par les populations et institutions locales des réformes nées des inspections littorales, elle constitue un volet de recherche en devenir tant il est complexe de la replacer dans le contexte des adhésions et résistances à l'emprise de l'État sur le littoral ${ }^{30}$. Les conséquences de l'inspection de Le Masson Du Parc (1723-1737) qui consistent en une régulation stricte des pratiques de pêche accroissant la misère des plus fragiles, pourraient servir de point de départ à une étude des évitements locaux et des résistances allant jusqu'à la révolte ouverte. L'après enquête constitue un temps historique important puisque s'y confrontent la vision par le haut de

28. Il s'agit du Titre IV, articles I à VIII du $4^{\mathrm{e}}$ Livre concernant la police des ports, côtes, rades et rivages de la mer.

29. Cf. Llinares, Sylviane, Autour de l'inspection Chardon, 1781-1785..., op. cit., sur l'enquête Chardon.

30. Le Bouedec, Gérard et ChAPPE, François, Pouvoirs et littoraux..., op. cit. 
l'État et la réalité des pratiques locales, un vécu au quotidien d'espaces où certains peinent à survivre. Cette différence d'appréciation provoquant des résistances et rébellions, chères à Jean Nicolas ${ }^{31}$, pourrait être interrogée dans le contexte global des postures adoptées face aux exigences de l'État, depuis la réponse incomplète, mensongère ou détournée à une demande d'informations jugée invasive, jusqu'à l'entrée en résistance concrète. Attitude appartenant au vaste ensemble des mécontentements populaires face aux réformes, elle pourrait trouver des prolongements, traces et résonances jusque dans les cahiers de doléances des cités portuaires.

Pour conclure cette présentation d'un projet en cours de réalisation partielle, rappelons que l'objectif en est de proposer la création d'une bibliothèque extensive des enquêtes accompagnée des outils de son exploitation, autrement dit un portail. Chacun pourra le nourrir comme il pourra s'en nourrir. Dans un premier temps, il servira à tester trois orientations de recherche concernant en premier lieu la mesure de la fiabilité documentaire de la source, puis de décrypter les temps et les rythmes de l'État face aux littoraux, enfin à saisir la perception de ces espaces par des acteurs aux intérêts divergents.

Pour ce faire ainsi que pour valider la pertinence et la richesse d'une analyse diachronique, les membres de l'équipe LETO proposent quelques approches publiées dans ce numéro des Annales de Bretagne et de Pays de l'Ouest et dans le suivant. Chaque contribution observe une portion du littoral en croisant les données de plusieurs enquêtes sur un thème ou un phénomène spécifique. Thierry Sauzeau a choisi les côtes de l'Aunis-Saintonge qu'il connaît particulièrement bien. Sébastien Martin et Christian Pfister concentrent leur attention sur une question fondamentale - celle de la fiabilité documentaire - dans leur article sur le cas des littoraux boulonnais et dunkerquois. Gilbert Buti a privilégié la frange maritime provençale comprise entre Martigues et Saint-Tropez, via Marseille et Toulon, pour décrypter l'état des lieux dressé pour l'État royal lors de l'enquête de 1665. Bernard Michon et David Plouviez ont choisi d'observer "les enquêtes comme indicateurs des existences portuaires. Le cas de l'estuaire de la Loire ". Enfin, Giulio Romero explique ce que pourrait être le portail envisagé sous la forme d'un SIH inspiré de l'outil SIG des géographes.

En partant des réflexions synthétiques de Giulio Romero et des cas de figure étudiés dans les différentes contributions, quelques résultats émergent concernant la méthodologie et le périmètre choisis pour l'analyse des enquêtes littorales ainsi que la pertinence de certains objectifs. La dimension diachronique de la ressource documentaire en paraît l'un des éléments majeurs. Elle permet de connaître l'histoire littorale d'un lieu, d'en mesurer les changements et les évolutions selon les époques,

31. NICOLAS, Jean, La rébellion française, mouvements populaires et conscience sociale, Paris, Seuil, 2002; AUBERT, Gauthier, Atlas de la rébellion française, projet ANR. 
éventuellement de les comparer à la situation actuelle afin d'en tirer des éléments informatifs. En ce sens, les remarques générales de Giulio Romero sont confirmées par celles de Thierry Sauzeau dans son analyse du cas du coureau d'Oléron. Concernant cet espace précis, il note que l'enquête de 1665 a donné lieu à une modeste mais véritable mission hydrographique revêtant tous les aspects de ce type de navigation : experts, mesures in situ, procès-verbaux. À partir de ce constat de fiabilité, il estime possible de se livrer à un travail de nature expérimentale incluant les mesures de 1665 dans le temps long. La trajectoire des fonds marins et des chenaux de navigation du coureau d'Oléron pourra ainsi être retracée depuis la fin du $\mathrm{XVII}^{\mathrm{e}}$ siècle jusqu'à la fin du Xx $\mathrm{x}^{\mathrm{e}}$ siècle. Par ailleurs, Thierry Sauzeau souligne un phénomène physique qui ressort de l'enquête de 1665 qu'il a confrontée à la cartographie d'époque et à la modélisation rétrospective des mouvements de marées. Le chenal central du coureau d'Oléron semble s'être approfondi à mesure que les berges s'envasaient. Un autre résultat de cette contribution sur le littoral entre Seudre et Charente permet d'avancer que la fermeture par envasement du chenal de Brouage fut accélérée par son abandon programmé au bénéfice de la création de Rochefort en 1666. Ces résultats confortent les principes de base adoptés par le dispositif LETO. Les enquêtes, analysées sur le temps long, dans un contexte documentaire enrichi d'autres ressources manuscrites et de la cartographie, passées au filtre des outils d'aujourd'hui, livrent des informations supplémentaires à celles qu'offre leur unique lecture isolée.

Dans son décryptage du littoral nord, la contribution de Sébastien Martin et Christian Pfister confirme d'autres options initiales. Outre le fait de concerner un local au particularisme fort lié, entre autres, à son rattachement tardif au royaume de France, le cas de Dunkerque, observé à plus d'un siècle d'écart par confrontation de deux enquêtes (1665 et 1782), permet aux auteurs de mettre en lumière la posture de réticence, voire de résistance, des élus locaux aux demandes de l'État monarchique, grâce en particulier à l'analyse textuelle dont ils soulignent la nécessaire utilisation.

Cette réflexion rejoint celles de Giulio Romero sur la réalisation d'un système d'information historique (SIH) qui implique une démarche commune concernant la transcription et l'unification des enquêtes, ainsi que de multiples précautions, afin que l'analyse textuelle puisse être menée d'un document à l'autre, d'une époque à l'autre ${ }^{32}$. Du fait de la nature même des sources, le plus souvent manuscrites, Giulio Romero indique clairement que la transcription ne peut pas s'en faire sous la forme d'une numérisation directe non corrigée (du type Google Books), qui lui apparaît dans ce cas comme un risque plus qu'un avantage et par ailleurs insuffisante aux

32. Il s'agit là d'une remarque désormais classique à propos de l'analyse groupée de textes. Nous renvoyons à la thèse récemment soutenue de Matthieu Quantin à l'École Centrale de Nantes sur l'approche multi-échelle et multi-critère de corpus textuels où il fait référence au programme HARUSPEX appliqué au corpus des biens inscrits sur la liste du patrimoine mondial. 
besoins d'un SIH. Il convient donc que les spécialistes transcrivent... et unifient les textes. L'enjeu est d'importance. Mot, nom de personne, toponymie, synonymie dans le temps et l'espace doivent être unifiés, y compris lorsqu'ils apparaissent dans les cartes ou l'iconographie. La question de l'unification grammaticale vers une langue modernisée semble aussi à retenir tandis que la sélection par mot isolé ou groupe de mots doit être résolue afin d'éviter les erreurs ${ }^{33}$.

Outre les aspects méthodologiques évoqués ici de façon synthétique, la construction d'un SIH doit permettre à tout chercheur extérieur à l'équipe initiale de s'y intégrer en utilisant les mêmes conditions d'expérience ou en variant ces conditions pour obtenir de nouveaux résultats. Cette obligation nécessite de conserver la documentation sous toutes ses formes - images, cartes, textes originaux, textes transcrits, commentaires d'images, bases de données, fichiers XML... - afin que tout chercheur trouve l'information recherchée dans le cadre exact dans lequel elle a été obtenue. Tout ce travail est le prix à payer pour obtenir un portail utilisable, facile d'accès, en rendant les enquêtes littorales visibles sur un même site, que ce soit sous leur forme native ou sous leur forme élaborée. Le meilleur moyen pour faciliter cet accès et la compréhension des enquêtes est d'en proposer des cas de traitement en cours ou finalisés avec les logiciels utiles, les personnes-ressources et les résultats. Les contributions qui suivent pourraient en être les premiers exemples, rejointes ultérieurement par d'autres travaux en cours ou à naître que l'intérêt d'un tel dispositif pourrait susciter, l'ensemble permettant une progression collective dans la connaissance des enquêtes littorales.

33. Par exemple, une sélection par mot isolé peut provoquer une confusion et une perte d'information. Dans l'expression " fromage blanc " si la sélection se fait sur le seul vocable " fromage ", elle crée un amalgame de deux catégories, un fromage et un dessert. Erreur et perte d'information fine. 
Annexe 1 - Liste des visites effectuées par Le Masson du Parc lors de son inspection des côtes du Ponant, 1723-1737

\begin{tabular}{|c|c|c|}
\hline Dates & lieux & Cotes CARAN \\
\hline $1723-1724$ & $\begin{array}{l}\text { Flandre, Picardie, Normandie. } \\
\text { Débute à Dunkerque. } \\
\text { Normandie : Du Tréport au Mont } \mathrm{S}^{\mathrm{t}} \text {-Michel }\end{array}$ & Marine C5 18 \\
\hline 1726 & Côtes nord de la Bretagne & Marine C5 20 \\
\hline $1727-1728$ & $\begin{array}{l}\text { Guyenne, Aunis, Saintonge. } \\
\text { Bayonne et Bordeaux } \\
\text { Marennes, La Rochelle, Sables-d'Olonne }\end{array}$ & $\begin{array}{l}\text { Marine C5 } 23 \\
\text { Marine C5 } 22\end{array}$ \\
\hline 1728 & $\begin{array}{l}\text { Bretagne sud } \\
\text { Nantes, Vannes, Quimper }\end{array}$ & Marine C5 21 \\
\hline Avril 1729 & $\begin{array}{l}\text { Côtes depuis le Blanc-Nez jusqu'au cap de } \\
\text { Barfleur }\end{array}$ & \\
\hline 1730 & $\begin{array}{l}\text { Côtes depuis Pontorson aux confins de la } \\
\text { Flandre (sens inverse de la visite précé- } \\
\text { dente) }\end{array}$ & \\
\hline 1733 & $\begin{array}{l}\text { Plusieurs inspections dans les Amirautés } \\
\text { de Quimper, Vannes, Nantes, Les Sables- } \\
\text { d'Olonne, La Rochelle }\end{array}$ & Marine C5 31 \\
\hline 1735 & De Brest au duché de Retz & Marine C5 32 \\
\hline 1736 & De Dunkerque à Caen & Id. \\
\hline 1737 & Id. & Id. \\
\hline
\end{tabular}

Verdier, successeur de Le Masson du Parc, poursuit les visites et tournées de 1738 à 1743 (Marine, C5 33 à 35).

\section{Annexe 2 - Procès-verbal de visite du 18 juillet 1665 signé Regnier Jansse (Français modernisé) (SHD Vincennes, SH 48, fo 251 rot $^{\circ}$ et $v^{o}$}

Nous Regnier Jansse ingénieur ordinaire du roi pour les digues et travaux de la mer certifions à tous ceux qu'il appartiendra d'avoir vu et visité toute la côte de la mer Méditerranée depuis le cap de la Franqui jusques au cap de Cette tout le long de laquelle nous avons trouvé si peu de profondeur d'eau comme aussi de battures de sables avec une côte basse lequel nous n'avons trouvé en aucune façon propre ni commode quelque aide que l'on y pourrait apporter par art et construction des grands ouvrages pour y bâtir des ports et havres pour la retraite des grands navires du roi même aussi au Grau de Palavas lequel est aussi tout comble de sable tant au-dedans de l'étang qu'au dehors.

Et ayant fait le long du rivage de la mer tout le tour de la montagne ou promontoire de Cette avons exactement remarqué que cet endroit est la [sic] plus profonde et moins incommode de sable de la susdite côte spécialement la côte orientale de ladite montagne où joignant icelle en y construisant un môle à l'endroit et suivant le moyen et modèle proposé par monsieur le Chevalier de Clerville lequel pourra 
servir de avant-port ou rade et donner lieu pour y bâtir un bon port et à l'abri d'icelle ouvrir un grand canal dedans l'étang de Thau par lequel on pourra faire entrer les barques pour y aller donner sonde dedans les lieux où il s'y trouve assez d'eau pour le tenir à flot toutes chargées. Mais que comme le courant de la mer ou ceux de l'étang pourraient embarrasser les entrées et la capacité de ce canal pour les sables que les uns et les autres charrieraient il serait nécessaire d'y faire une écluse de vingt quatre pieds d'ouverture à soixante dix toises de la mer.

Par le moyen de laquelle l'agitation y fut arrêtée de part et d'autre et les dits sables empêchés d'y couler faute de l'impulsion dont ils ont ordinairement besoin pour les conduire.

En outre ajoute à tout cela que bien que l'on pourrait estimer que ledit canal fut suffisant pour introduire des barques dedans l'étang de Thau et la susdite écluse pour en empêcher l'ensablement je crois et soutiens que l'on ne s'en peut pas servir utilement ni même en faire la dépense sans y joindre en même temps la susdite rade ou avant-port à la partie susdite du cap qui regarde directement le levant qui se pourrait faire au-devant de son entrée en attachant une jetée longue d'au moins trois cents toises : sans quoi il ne faut penser en aucune façon qu'on doit faire le canal susdit par la raison que s'il n'y avait point un abri assuré au-devant de l'ouverture du susdit canal, les barques qui y viendraient de haute mer n'y pourraient point entrer que avec grand périls et dangers d'être brisées contre la côte et rochers. Fait à Montpellier le $18^{\mathrm{me}}$ juillet 1665 .

Regnier Jansse 


\section{Carte de l'embouchure de la Gironde, fin XVII siècle}

(SHD Vincennes, SH 79)

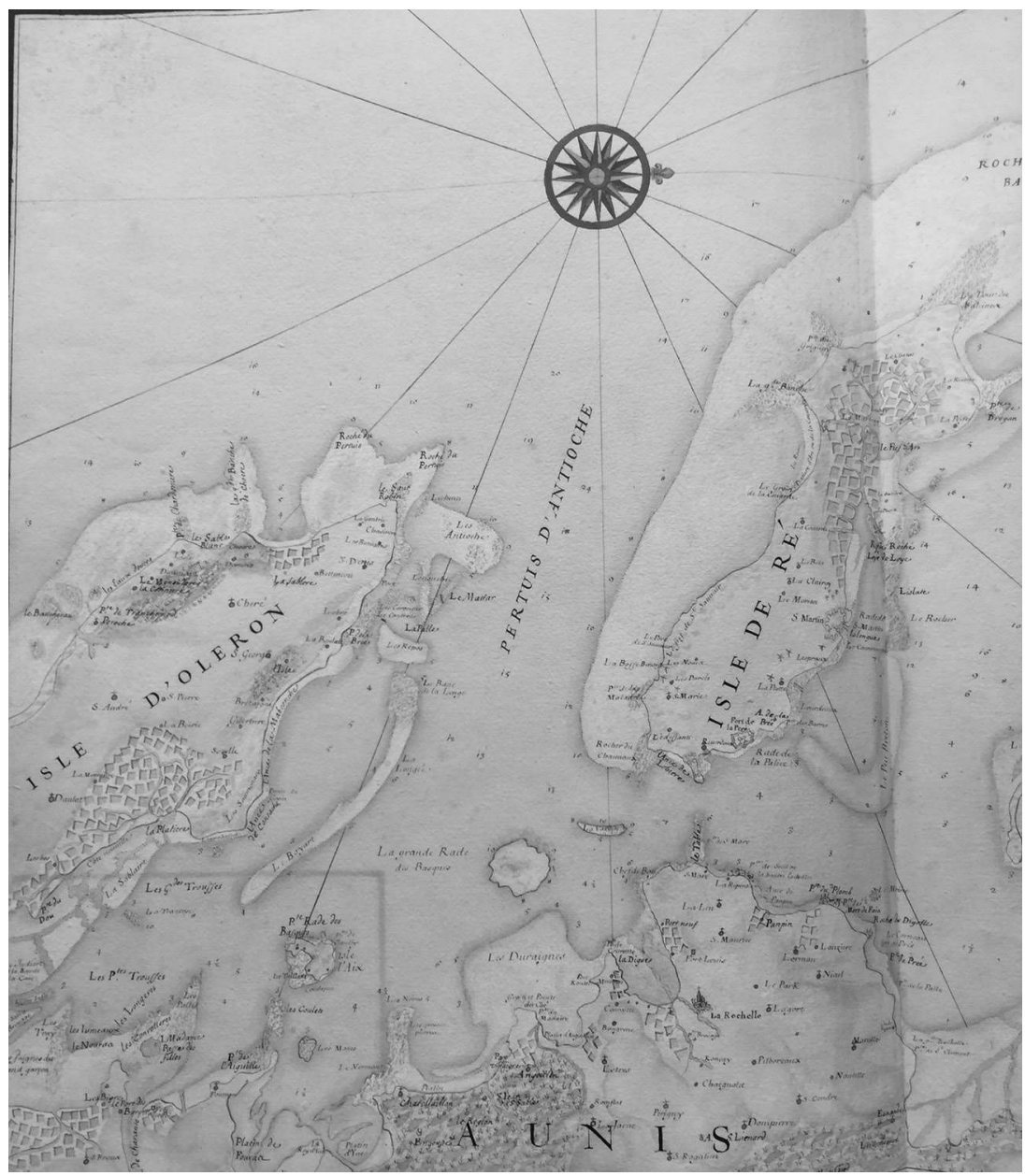


Un dispositif test sur les enquêtes littorales

\section{Écluse de Régnier Jansse}

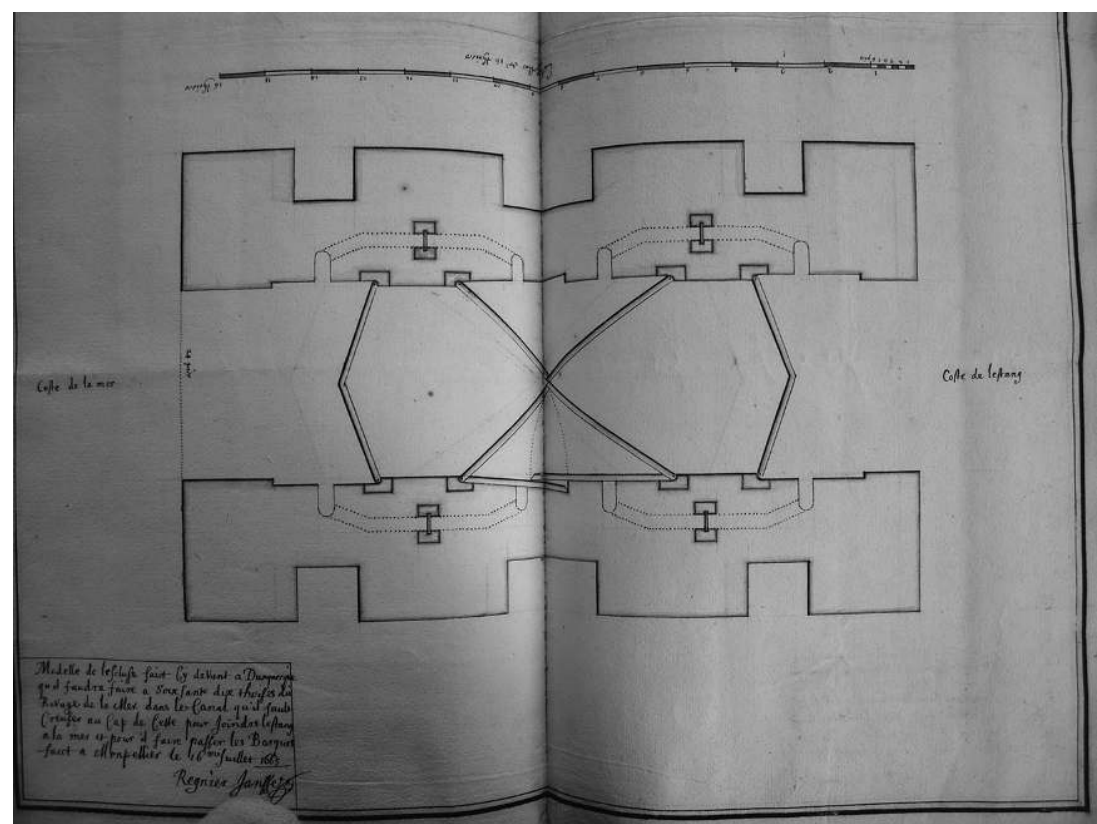




\section{RÉSUMÉ}

Une petite équipe de chercheurs intéressés par le phénomène des enquêtes littorales à l'époque moderne a réfléchi à la construction d'un portail, un Système Informatique Historique qui soit à la fois une bibliothèque extensive des enquêtes et un faisceau d'outils nécessaires à leur exploitation. Ce dispositif est présenté ici et décrit dans le contexte historiographique d'aujourd'hui, avec ses objectifs, ses modalités et ses tests de faisabilité, ces derniers faisant l'objet de quatre contributions publiées à la suite de cette introduction programmatique. La particularité du dispositif LETO consiste à considérer la continuité séculaire du phénomène de l'enquête littorale et à s'interroger sur la profondeur de la mémoire d'État, en convoquant et confrontant une dizaine d'enquêtes dans leurs modalités de construction et d'exécution, leurs contenus informatifs et leurs conséquences autant pour l'État qui les commandite que pour les acteurs littoraux qui les subissent et y réagissent.

\section{ABSTRACT}

A small team of researchers interesting in the phenomenon of early modern coastal surveys has sought to create a gateway, a computerised historical system that would simultaneously be a comprehensive register of surveys and a collection of tools necessary for their analysis. This package is presented here and described in the contemporary historiographical context along with the project's objectives, its modalities and the feasibility study - these latter elements having been the object of articles that are published immediately after this programmatic introduction. The particularity of the LETO project is that it seeks to understand the secular continuity of the phenomenon of costal surveys and compare the surveys, the details of their organisation and execution, the information they contain and their impact on the State that commissioned them and on the coastal men and women who endured and reacted to them. 